\title{
Downregulation of Long Noncoding RNA TUG1 Attenuate MTDH /NF-KB/IL-1 $\beta$ mediated inflammatory damage via Targeting miR-29b-1-5p After Spinal cord ischemia reperfusion in rats
}

Hui Jia

China Medical University First Hospital

Zhe Li

China Medical University First Hospital

Bo Fang

China Medical University First Hospital

\section{Yi Chang}

China Medical University First Hospital

\section{Yongjian Zhou}

China Medical University First Hospital

Hong Ma ( $\square$ mahong54665466@126.com )

China Medical University First Hospital https://orcid.org/0000-0002-7253-5413

\section{Research}

Keywords: Spinal cord ischemia reperfusion injury, Neuroinflammation, Blood-spinal cord barrier, Astrocytes, TUG1, miR-29b-1-5p, MTDH

Posted Date: February 26th, 2020

DOI: https://doi.org/10.21203/rs.2.24618/v1

License: (c) (i) This work is licensed under a Creative Commons Attribution 4.0 International License. Read Full License 


\section{Abstract}

Background: Spinal cord ischemia reperfusion (IR) is associated with an inflammatory response. The long non-coding RNA (IncRNA) taurine upregulated gene 1 (TUG1) and microRNA-29b (miR-29b) family are frequently dysregulated in neuro-ischemic diseases. However, their potential roles in spinal cord IR injury (IR) are unknown.

Methods: A spinal cord IR model was established in rats by14-minute occlusion of aortic arch. The aberrant miRNAs were identified by microarray analysis, and qRT-PCR was used to validate the IncRNA and microRNA levels. The motor function of the differentially-treated animals was assessed by Tarlov scores, and the leakage of Blood-spinal cord barrier (BSCB) was measured in terms of the extravasation of Evans blue (EB) dye. The expression levels of different proteins were analyzed by Western blotting and immunofluorescence. The interaction between TUG1 and miR-29b-1-5p, TRIL and miR-29b-1-5p, and MTDH and miR-29b-1-5p were determined using bioinformatics programs and the dual-luciferase reporter assay.

Results: MiR-29b-1-5p was significantly downregulated and TUG1 was upregulated in the spinal cord of rats after IR. In addition, TRIL and MTDH protein levels were also significantly increased after IR. MTDH was predicted as a target of miR-29b-1-5p and its knockdown downregulated NF-KB and IL-1 $\beta$ levels. In addition, a direct interaction was observed between TUG1 and miR-29b-1-5p, and knocking down TUG1 upregulated the miRNA. Furthermore, overexpression of miR-29b-1-5p or TUG1 knockdown alleviated BSCB leakage and improved hind-limb motor function, and downregulated MTDH and its downstream pro-inflammatory cytokines. Suppression of miR-29b-1-5p reversed the neuroprotective effect of TUG1 knockdown, restored the levels of MTDH/ NF-KB/IL-1 $\beta$ and activated astrocytes.

Conclusion: Downregulation of TUG1 alleviated MTDH/NF-KB/IL-1 $\beta$ pathway-mediated inflammatory damage after IR by targeting miR-29b-1-5p. Keywords: Spinal cord ischemia reperfusion injury, Neuroinflammation, Blood-spinal cord barrier, Astrocytes, TUG1, miR-29b-1-5p, MTDH

\section{Background}

Spinal cord ischemia reperfusion injury (IR) is a common complication of thoracoabdominal aortic aneurysm surgery [1], and can progress to paralysis [2]. However, the underlying molecular mechanisms are poorly understood. Therefore, it is essential to identify the factors involved in regulating spinal cord IR in order to improve patient prognosis. MicroRNAs (miRNAs) are $~ 22$ nucleotides long small non-coding RNAs [3] that repress gene expression by binding to the 3'-untranslated region (UTR) of the target mRNAs. Several miRNAs have been identified as potential therapeutic targets in tumors [5], infections [6], immune disorders [7], cardiovascular diseases [8], as well as neurodegenerative diseases [9] like spinal cord IR [10]. MiR-29 is involved in the pathogenesis of various neurological diseases $[11,12]$ and nerve injury.

The NF-kB mediated inflammatory signaling pathway also plays a vital role in aggravating spinal cord IR [13]. In addition, the TLR4 interactor with leucine-rich repeats (TRIL), a ligand of TLR4, is highly expressed 
in the spinal cord after IR [14]. Likewise, metadherin (MTDH) or astrocyte elevated gene 1 is upregulated in various tumors [15] and neurodegeneration diseases [16], and triggers the NF-KB pathway [17], although its potential role in spinal cord IR is unknown. Long non-coding RNAs (IncRNAs) are a class of non-coding transcripts longer than 200 bases [18] that regulate gene expression by targeting the miRNAs as endogenous RNAs (ceRNAs) [19]. Taurine-upregulated gene 1 (TUG1) promotes the normal development of photoreceptors of retina [20], and is aberrantly expressed during neurodegeneration [21, 22] and inflammation [23]. In our previous study, we found that knocking down TUG1 inhibited NF-KB pathway-induced inflammatory damage in spinal cord IR [14].

In our study, miR-29b-1-5p was substantially downregulated after spinal cord IR in a rat model. We found a regulatory network involving IncRNA-TUG1, microRNA-29b-1-5p and the MTDH/NF-KB inflammatory pathway in spinal cord IR.

\section{Materials And Methods}

\section{Establishment of rat spinal cord IR model}

The IR model was induced as previously reported [14] in 8-weeks old male Sprague-Dawley rats. Briefly, the descending aorta was cross-clamped distal to the left subclavian artery following left thoracotomy in order to obstruct arterial flow to the spinal cord. The occlusion was removed 14 minutes later to restore perfusion. The sham-operated rats underwent thoracotomy without arterial occlusion. All animal experiments were approved by the China Medical University Animal Care and Use Committee (2017105).

\section{MiRNA microarray analysis}

Total RNA was extracted from the lumbar vertebra segments 4-6 (L4-6) using Trizol reagent as previously reported [24], and the miRNAs were analyzed by Affymetrix microarrays (CapitalBio Corp). Hierarchical clustering was performed to detect the aberrantly expressed miRNAs using MEV software (version 4.6; TIGR, Microarray Software Suite4, Boston, United States).

\section{Intrathecal Injection}

The animals were intrathecally injected with the synthetic miRs or siRNAs in the L4-L6 segments 3 days before IR induction as previously reported [14]. The hind-limb motor function of the rats was observed after intrathecal injection, and only those with normal movement were selected for the subsequent

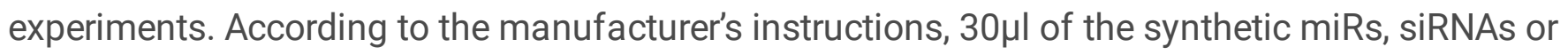
corresponding controls (GenePharma, Shanghai, China) was infused intrathecally along with Lipofectamine 2000 (Invitrogen, MA, USA). The siRNA sequences were as follows:

miR-29b-1-5p mimic: 5' -UUUCAUAUGGUGGUUUAGAUUU-3'

miR-29b-1-5p inhibitor: 5'-AAAUCUAAACCACCAUAUGAAA-3' 
Negative control: 5' -CAGUACUUUUGUGUAGUACAA-3'.

si- MTDH -1: CCUGAAGAUGAAGUUGUUATT;

si- MTDH -2: GCAGUCUGCUUGGACUCAATT;

si- MTDH -3: CCAGGAGCCGAUUUCUAAUTT.

Scramble siRNA (si-NC): 5' -CUCUGAACCCUAAGGCCAATT-3'.

si-TUG1-1: GCAGUAAUUGGAGUGGAUATT;

si-TUG1-2: GCAGAUAUUCUGACCCAUUTT;

si-TUG1-3: CCAUCUCACAAGGCUUCAATT.

si-NC: 5'-UUCUCCGAACGUGUCACGUTT-3'

\section{Quantitative real-time polymerase chain reaction}

Total RNA of L4 to L6 spinal cord segments was isolated by Trizol reagent (Takara, Otsu, Japan) as previously reported [25]. TaqMan MicroRNA Assay Kit (Applied Biosystems) was used for quantifying miR-29b-1-5p with the following primer: forward 5'-CGCGCGTTTCATATGGTGGTTTAGATTT-3'. U6 was used as the internal control (forward 5'-CTCGCTTCGGCAGCACA-3'). For TUG1 expression analysis, PrimeScript RT reagent Kit with gDNA Eraser (Takara) was used for reversed transcription, and GAPDH was used as the internal control. The primer sequences (Sangon Biotech, Shanghai, China) were as follows: TUG1: forward 5'-TGCCACCAGCACTGTCACT-3' and reverse 5'-ACGGTCCAGGTGAATGAACA -3'; GADPH: forward 5'-GGGGCTCTCTGCTCCTCCCTG-3' and reverse 5'-AGGCGTCCGATACGGCCAAA-3'. Relative expression levels were measured using the $2^{-} \triangle \triangle \mathrm{Ct}$ method.

\section{Western Blotting}

Western blotting was performed as previously described [26] using primary antibodies against TRIL (1:500; Santa Cruz), MTDH (1:1000; Proteintech), NF-KB p65 (1:1000; Abcam), IL-1 1 (1:1000; Abcam) and polyclonal anti- $\beta$-actin (1:500; Abcam), and the corresponding secondary antibodies (Bioss, Beijing, China).

\section{Immunofluorescence}

The in situ expression of MTDH and the astrocytic marker GFAP was analyzed as described previously [27]. The fixed spinal cord was sectioned into $10 \mathrm{~mm}$-thick slices, and probed with rabbit anti-MTDH (1:100, Proteintech), rabbit anti-GFAP (1:800, ServiceBio) and rabbit anti-DAPI (1:800, ServiceBio) primary antibodies, followed by Cy3-labeled goat anti-rabbit lgG secondary antibody (1:500, ServiceBio). The stained sections were observed under the Leica TCS SP2 laser scanning microscope. 


\section{Neurological Assessment}

Hind limb motor function was assessed $12 \mathrm{~h}$ after IR by two observers blinded to the experimental grouping using the Tarlov scale [10]. The locomotor function was scored as follows: 0 - lower limb function deficiency, 1 - visible lower limb movement but weak against gravity, 2 - occasional lower limb movement and against gravity but inability to stand, 3 - abnormal standing posture and walk, and 4 completely normal movement.

\section{Assessment of Evans Blue Extravasation}

Evans blue (EB) fluorescence was used to assess blood-spinal cord barrier (BSCB) leakage as previously described [14]. Briefly, EB was injected into the rats 1h before sacrifice via their tail vein. The L4-L6 spinal cord segments were later dissected and fixed in paraformaldehyde (Beyotime Biotechnology), cut into $10 \mu \mathrm{m}$-thick sections, and viewed under a fluorescence microscope (Olympus, Melville, NY) using the green filter.

\section{Luciferase assay}

The binding site of miR-29b-1-5p in TUG1(NR_130147) was predicted by RNAhybrid 2.2 (https://bibiserv.cebitec.uni-bielefeld.de/rnahybrid/). Potential binding sites of miRNA-29b-1-5p in MTDH (NM_133398) and TRIL (NM_001034010) were predicted by TargetScan (http://www.targetscan.org/).

The pGL3 firefly luciferase reporter plasmid sequences were as follows:

wild-type (WT) MTDH (3'UTR: 5'-CAAAGGGAAAGTTAATTTATGAAAT-3') and mutated (MT) MTDH (3'UTR:5'- AAAGGGAAAGTTAATTGCGTCCAT-3')

wild-type (WT) TRIL (3'UTR: 5'-AAGCTACCTGAGACAATATGAAG-3')

or mutated (MT) TRIL (3'UTR: 5'-AAGCTACCTGAGACACGCGTCCG-3')

wild-type (WT) TUG1 (3'UTR: 5'-CATCTTTACCACCATGGTGAT-3')

or mutated (MT) TUG1 (3'UTR:5'-ACGAGGGCAACAACGTTGTCG-3'). The HEK293 cells were cotransfected with the wild-type or mutant oligonucleotides and miR-29b-1-5p or negative control. Luciferase activity was measured by Reporter Assay Kit (Promega Corp., Madison, WI, USA), and normalized to that of Renilla luciferase.

\section{Statistical Analysis}

All data were expressed as mean \pm standard error (SEM) and SPSS software (version 22.0, SPSS, Inc., USA) was used for statistical analysis. Student t-test or two-way analysis of variance (ANOVA) were used to compare two or multiple groups respectively. The correlation between TUG1 and miR-29b-1-5p expression levels was assessed by Spearman correlation test. Kruskal-Wallis test with Bonferroni 
correction was used to assess Tarlov scores, and $p<0.008, p<0.005$ and $p<0.003$ were considered statistically significant for 4,5 and 6 groups. For other analyses, $P<0.05$ was regarded as statistically significant.

\section{Results}

\section{MicroRNA expression profiles in rats spinal cord after IR}

A total of 141 miRNAs were identified in the microarray, of which one was upregulated (up to $>2$ fold) and 19 were downregulated ( $<0.5$ fold) after IR (Figure 1a, Table 1). Compared to the sham group, miR-29b-1$5 p$ was seriously downregulated after IR in a time-dependent manner and reached the minimum levels after $12 \mathrm{~h}$ (Figure 1b). Therefore, subsequent experiments were conducted at the $12 \mathrm{~h}$ time point.

Table 1. Representative aberrant miRNAs after IR in rat spinal cord.

\begin{tabular}{ll}
\hline \multicolumn{1}{c}{ miRNA } & \multicolumn{1}{c}{ Fold-change } \\
\hline $\begin{array}{c}\text { Upregulated miRNAs } \\
\text { rno-miR-3568 }\end{array}$ & 3.57657193002397 \\
downregulated miRNAs & \\
rno-miR-872-3p & 0.657657703867661 \\
rno-miR-338-3p & 0.261863311 \\
rno-miR-181a-5p & 0.537212 \\
rno-miR-328b-3p & 0.537212 \\
rno-miR-702-3p & 0.482823476 \\
rno-miR-410-5p & 0.41488511 \\
rno-miR-181a-1-3p & 0.422475327 \\
rno-miR-488-3p & 0.327406346 \\
rno-miR-221-3p & 0.263389068 \\
rno-miR-181b-1-3p & 0.326677303 \\
rno-miR-135b-5p & 0.482509558 \\
rno-miR-129-1-3p & 0.468857651 \\
rno-miR-9a-5p & 0.390349615 \\
rno-miR-29b-1-5p & 0.379478948 \\
rno-miR-196b-5p & 0.385921325 \\
rno-miR-582-3p & 0.451759689 \\
rno-miR-879-3p & 0.353418211 \\
rno-miR-365-3p & 0.454538101 \\
rno-miR-370-5p & 0.321362523 \\
\hline
\end{tabular}

\section{MiR-29b-1-5p mimic alleviated the neurological deficit after IR}

In order to elucidate the neurological function of miR-29b-1-5p, the spinal cord IR-modeled rats were injected with the mimic. As shown in Figure 2a, mimic-29b-1-5p significantly increased the miRNA levels after IR compared to the untreated and NC-29b-1-5p-treated groups. Furthermore, mimic-29b-1-5p also restored the Tarlov scores of locomotor function that were decreased significantly due to IR, whereas the NC-29b-1-5p had no discernible effect (Figure 2b). Consistent with this, ectopic level of miR-29b-1-5p also significantly attenuated the IR-induced BSCB leakage and EB extravasation (Figure 2c, d). These results point to a protective role of miR-29b-1-5p in spinal cord IR. 


\section{MiR-29b-1-5p targets the MTDH/NF-kB axis after spinal cord IR}

To further explore the above surmise, we predicted the targets of miR-29b-1-5p, and identified complementary 3'-UTR binding sites in TRIL and MTDH (Figure 3a, b). Consistent with this, both proteins were overexpressed after IR (Figure 3c-e). Furthermore, knocking down MTDH with specific siRNAs (Figure 4a, b) significantly downregulated NF-KB p65 and IL-1 $\beta$ after IR (Figure 4c-e). The mimic-29b-1-5p also significantly decreased MTDH, NF-KB p65 and IL-1 $\beta$ in the rats after IR (Figure 5a, e), but did not affect TRIL protein levels. Luciferase reporter assay further showed that the wild-type MTDH 3'-UTR was suppressed by the miR-29b-1-5p precursor (Figure 5f), whereas its repressive action was negated if the 3'UTR of MTDH carried a mutated binding site for the miRNA. In contrast, neither wild-type nor mutant TRIL-3'-UTR was affected by the miR-29b-1-5p precursor (Figure 5g). Taken together, miR-29b-1-5p inhibits the NF-KB pathway by directly targeting MTDH.

\section{The IncRNA TUG1 is the ceRNA of miR-29b-1-5p}

To determine the potential regulatory role of TUG1 on miR-29b-1-5p, we predicted the binding sites of the latter on TUG1 (Figure 6a). Furthermore, TUG1 was upregulated in a time-dependent manner and peaked $12 \mathrm{~h}$ post-IR (Figure $6 \mathrm{~b}$ ), and correlated negatively with miR-29b-1-5p level (Figure 6c). Consistent with this, knocking down TUG1 (Figure 6d) significantly increased miR-29b-1-5p expression $(P<0.05)$ after IR. In addition, the luciferase activity of the reporter under the wild-type TUG1-3'-UTR was suppressed by miR29b-1-5p precursor, while mutation in the binding site eliminated its repressive effect (Figure 6f). Taken together, TUG1 acts as ceRNA for miR-29b-1-5p and both share a negative regulatory relationship.

\section{TUG1 aggravates spinal cord IR by targeting miR-29b-1-5p and activating the MTDH/NF-KB/IL-1 $\beta$ pathway}

To further establish the biological relevance of TUG1 in neurological injury post spinal cord IR, we treated the modeled rats with si-TUG1. As shown in Figure 7a, si-TUG1 significantly increased the Tarlov scores after IR along with attenuating BSCB extravasation (Figure 7b), and significantly downregulated MTDH, NF-KB and IL-1 $\beta$ after IR (Figure 8a-d). In line with our findings so far, silencing TUG1 upregulated miR29b-1-5p during IR, which was neutralized by the specific miRNA inhibitor $(P<0.05$; Figure 9a).

Furthermore, suppression of miR-29b-1-5p reversed the ameliorative effects of TUG1 knockdown on Tarlov scores (Figure 9b) and BSCB leakage (Figure 9c,d), thereby highlighting the regulatory role of the miR-29b-1-5p/TUG1 axis in spinal cord IR. Mechanistically, inhibition of miR-29b-1-5p restored the levels of MTDH, NF-KB p65 and IL-1 $\beta$ after IR in the presence of TUG1 knockdown (Figure 10a-f). Finally, IR in the spinal cord significantly increased the expression of the astrocyte marker GFAP, which was decreased upon TUG1 knockdown, and restored by the inhibition of miR-29b-1-5p (Figure 11a, b). We can conclude therefore that TUG1 plays a pathological role in spinal cord IR by targeting miR-29b-1-5p, which activates the pro-inflammatory NF-kB pathway.

\section{Discussion}


We identified a novel TUG1/miR-29b-1-5p/MTDH/ NF-KB/IL-1 $\beta$ regulatory axis in a rat model of spinal cord IR. MiR-29b-1-5p exerted a protective role in terms of restoring lower limb motor function and alleviating BSCB leakage after IR by targeting MTDH, and blocking the downstream pro-inflammatory NFKB/IL-1 $\beta$ pathway. The IncRNA TUG1 was highly expressed after IR and acted as a ceRNA for miR-29b-1$5 p$. It suppressed miR-29b-1-5p and aggravated IR, most likely by restoring MTDH/NF-KB/IL-1 $\beta$ activity.

Several miRNAs are aberrantly expressed during spinal cord IR, and may act as protective or pathological factors. For example, increased levels of miR-27a attenuated IR-related damage in the spinal cord by targeting the TLR4 inflammatory pathway [24], while miR-199a-5p also exerted a protective effect by blocking ECE1-mediated apoptosis signaling [28]. In contrast, inhibiting miRNA-124 protected rats against spinal cord IR by inducing mitophagy [29]. Previous reports on spinal cord IR microarrays [30, 31] have also identified miR-29, which is involved in neurological diseases such as stroke [11], Huntington's disease [33] Parkinson's disease [32] and Alzheimer's disease [12], and was significantly downregulated in the hippocampal neurons of a rat model of brain hypoxia [34]. We have firstly shown miR-29b-1-5p level was markedly decreased after spinal cord IR, and may therefore play a protective role. Indeed, intrathecal administration of the miR-29b-1-5p mimic improved motor function and decreased blood-spinal barrier (BSCB) leakage in the rats after IR.

BSCB integrity is vital to maintaining homeostasis between capillaries and the spinal cord [35], and is disrupted during an inflammatory response. The inflammatory cytokines disintegrate the tight junction proteins, which increases vascular permeability and exposes the spinal cord to circulating pathogens, thereby increasing the risk of neuronal damage [35]. IR-induced inflammation upregulates the matrix metalloproteinase-9 (MMP-9) and downregulates the $x x$ protein [36]. In addition, intrathecal injection of TLR4 inhibitors attenuated BSCB leakage and inflammatory responses [37]. Therefore, we hypothesized that miR-29b-1-5p exerted a protective function by inhibiting the inflammatory responses.

MiRNAs inhibit gene level by binding to the 3'-UTR of target mRNAs [4]. We identified the inflammationrelated proteins TRIL and MTDH as the putative targets of miR-29b-1-5p. In a previous research, we suggested that TRIL was upregulated in the spinal cord after IR and its knockdown reduced the levels of NF-kB-dependent cytokines. Similar results were seen in the present study as well, although we could not establish a direct interaction between the the 3' UTR of TRIL and miR-29b-1-5p. MTDH is overexpressed in multiple tumors and correlates with increased tumor progression, invasion and metastasis, as well as poor prognosis [15]. In addition, MTDH is also involved in central nervous system diseases such as Huntington's disease, HIV-related dementia and migraine [38]. MTDH silencing inhibited proliferation and migration of Schwann cells in distal sciatic nerve injury [39]. It is known to trigger the NF-kB pathway [17], and high levels of MTDH in glioma promotes the release of pro-inflammatory cytokines [40]. We found that IR significantly upregulated MTDH, and its knockdown decreased NF-KB and IL-1 $\beta$ expressions, indicating that the NF-KB pathway lies downstream of MTDH in IR. Furthermore, the dual-luciferase reporter assay established a direct interaction between miR-29b-1-3p and MTDH, and forced expression of the former blocked the MTDH/NF-KB/IL-1 $\beta$ pathway. Thus, miR-29b-1-5p assuages IR by inhibiting the inflammatory pathway via targeting $\mathrm{MTDH}$. 
We further identified the IncRNA TUG1 as a putative upstream factor of miR-29b-1-5p. TUG1 is upregulated in neurological [21,22] and ischemic diseases [41], inflammation [42], as well as in animal models of cerebral ischemia [43] and oxygen-glucose deprivation (OGD) injury [44]. Furthermore, overexpression of TUG1 exacerbated hypoxia-induced myocardial injury in cardiomyocytes [45], and the TUG1/miR-214/HSP27 pathway is involved in SCIRI [46]. We previously detected high expression levels

of TUG1 after IR [14], and found that TUG1 knockdown neutralized the neurological damage and BSCB leakage by inhibiting the TLR4/NF-KB inflammatory pathway. In the present study as well, TUG1 silencing exerted a neuroprotective effect by downregulating MTDH/NF-KB/IL-1 $\beta$ signaling. In addition, miR-29b-1$5 p$ level was negatively related to that of TUG1, and ablation of TUG1 upregulated miR-29b-1-5p. The luciferase reporter assay further confirmed binding between the two, strongly indicating that TUG1 acts a ceRNA to miR-29b-1-5p. Several studies have reported that IncRNAs suppress miRNA expression by functioning as ceRNAs [47]. For example, the IncRNA MALAT1 protected the spinal cord from IR-induced damage by targeting miR-204 [48]. Similarly, ablation of CasC7 promoted apoptosis in the ischemic spinal cord [49] via upregulation of miR-30c. We therefore speculated a similar regulatory interplay between TUG1, miR-29b-1-5p and MTDH during spinal cord IR. Indeed, the miR-29b-1-5p inhibitor largely reversed the neuroprotective effect of TUG1 knockdown, and restored the MTDH/NF-KB/IL-1 $\beta$ pathway.

Astrogliosis is a characteristic feature of central nervous system diseases, and accompanied by increased expression of the glial fibrillary acidic protein (GFAP) [50]. IR also activates the astrocytes, which can be attenuated by anti-inflammatory drugs [13]. In the present study, the astrogliosis induced by spinal cord IR was decreased by TUG1 knockdown and restored by the miR-29b-1-5p inhibitor. Previous reports have shown that MTDH colocalizes with the GFAP + reactive astrocytes in the injured area [51], and its knockdown suppresses astrocytes migration and proliferation [16]. MTDH-overexpressing astrocytes correlate significantly with excitotoxic neuronal damage [38]. Our data indicate that the TUG1/ miR-29b-1-5p/MTDH pathway mediates the inflammatory response of astrocytes during spinal cord IR. Furthermore, since an IncRNA can bind to multiple miRNAs [47], and miRNAs also have multiple target [52], it is feasible that TUG1 regulates TRIL directly or via other miRNAs, which is another therapeutic possibility that can be explored in future.

\section{Conclusion}

To summarize, the interaction between miR-29b-1-5p and TUG1 regulates the MTDH/NF-KB/IL-1 $\beta$ pathway in the ischemic spinal cord, and is therefore a potential therapeutic target for spinal cord ischemia injury.

\section{Abbreviations}

Spinal cord IR: Spinal cord ischemia reperfusion; IncRNA: long noncoding RNA; TUG1: taurine upregulated gene 1; miRNAs: MicroRNAs; miR-29b :microRNA-29b; BSCB: Blood-spinal cord barrier; EB: Evans blue; MTDH: metadherin; TRIL: TLR4 interactor with leucine- rich repeats; GFAP: glial fibrillary acidic protein; $3^{\prime}$-UTR : $3^{\prime}$-untranslated region 


\section{Declarations}

Acknowledgements

Not applicable

\section{Funding}

This work was supported by the National Natural Science Foundation of China (No. 81771342 and 81971152)

\section{Availability of data and materials}

The datasets obtained of this article are available upon request.

\section{Authors' contributions}

$\mathrm{HJ}, \mathrm{BF}$ and HM designed the stdy, HJ, ZL and YJZ constructed the IR model, HJ, ZL and YJZ performed the experiments, $\mathrm{HJ}$ and $\mathrm{YC}$ analyzed the data, and $\mathrm{HJ}$ and $\mathrm{HM}$ wrote and revised the manuscript. All authors have reviewed and approved the manuscript.

\section{Ethics approval}

All animal experiments were approved by the Ethics Committee of China Medical University.

\section{Consent for publication}

Not applicable.

\section{Competing interests}

All authors declare that they have no competing interests.

\section{References}

1. Wynn MM, Acher CW.A modern theory of spinal cord ischemia/injury in thoracoabdominal aortic surgery and its implications for prevention of paralysis.J Cardiothorac Vasc Anesth.2014;28:108899.

2. Hu J, Yu Q, Xie L, Zhu H.Targeting the blood-spinal cord barrier: A therapeutic approach to spinal cord protection against ischemia-reperfusion injury.Life Sci.2016;158:1-6.

3. Hanin G, Yayon N, Tzur Y, Haviv R, Bennett ER, Udi S, et al.miRNA-132 induces hepatic steatosis and hyperlipidaemia by synergistic multitarget suppression.Gut.2018;67:1124-34.

4. Svoboda P.A toolbox for miRNA analysis.FEBS Lett.2015;589:1694-701. 
5. Mott JL, Kurita S, Cazanave SC, Bronk SF, Werneburg NW, Fernandez-Zapico ME.Transcriptional suppression of mir-29b-1/mir-29a promoter by c-Myc, hedgehog, and NF-kappaB.J Cell Biochem.2010;110:1155-64.

6. Castro FL, Geddes VEV, Monteiro FLL, Goncalves R, Campanati L, Pezzuto P, et al.MicroRNAs 145 and 148a Are Upregulated During Congenital Zika Virus Infection.ASN Neuro.2019;11:1759091419850983.

7. Shikano S, Gon Y, Maruoka S, Shimizu T, Kozu Y, lida Y, et al.Increased extracellular vesicle miRNA466 family in the bronchoalveolar lavage fluid as a precipitating factor of ARDS.BMC Pulm Med.2019;19:110.

8. Jing R, Zhong QQ, Long TY, Pan W, Qian ZX.Downregulated miRNA-26a-5p induces the apoptosis of endothelial cells in coronary heart disease by inhibiting PI3K/AKT pathway.Eur Rev Med Pharmacol Sci.2019;23:4940-47.

9. Sadlon A, Takousis P, Alexopoulos P, Evangelou E, Prokopenko I, Perneczky R.miRNAs Identify Shared Pathways in Alzheimer's and Parkinson's Diseases.Trends Mol Med.2019.

10. Li XQ, Fang B, Tan WF, Wang ZL, Sun XJ, Zhang ZL, et al.miR-320a affects spinal cord edema through negatively regulating aquaporin- 1 of blood-spinal cord barrier during bimodal stage after ischemia reperfusion injury in rats.BMC Neurosci.2016;17:10.

11. Jolana L, Kamil D.The Role of microRNA in Ischemic and Hemorrhagic Stroke.Curr Drug Deliv.2017;14:816-31.

12. Gupta P, Bhattacharjee S, Sharma AR, Sharma G, Lee SS, Chakraborty C.miRNAs in Alzheimer Disease - A Therapeutic Perspective.Curr Alzheimer Res.2017;14:1198-206.

13. Li XQ, Lv HW, Tan WF, Fang B, Wang H, Ma H.Role of the TLR4 pathway in blood-spinal cord barrier dysfunction during the bimodal stage after ischemia/reperfusion injury in rats.J Neuroinflammation.2014;11:62.

14. Jia H, Ma H, Li Z, Chen F, Fang B, Cao X, et al.Downregulation of LncRNA TUG1 Inhibited TLR4 Signaling Pathway-Mediated Inflammatory Damage After Spinal Cord Ischemia Reperfusion in Rats via Suppressing TRIL Expression.J Neuropathol Exp Neurol.2019;78:268-82.

15. Dhiman G, Srivastava N, Goyal M, Rakha E, Lothion-Roy J, Mongan NP, et al.Metadherin: A Therapeutic Target in Multiple Cancers.Front Oncol.2019;9:349.

16. Vartak-Sharma N, Ghorpade A.Astrocyte elevated gene-1 regulates astrocyte responses to neural injury: implications for reactive astrogliosis and neurodegeneration.J Neuroinflammation.2012;9:195.

17. Hong R, Wang K, Shi H.Astrocyte elevated gene-1 promotes inflammation and invasion of fibroblastlike synoviocytes in rheumatoid arthritis.Tissue Cell.2017;49:672-79.

18. Wen J, Yang CY, Lu J, Wang XY.Ptprj-as1 mediates inflammatory injury after intracerebral hemorrhage by activating NF-kappaB pathway.Eur Rev Med Pharmacol Sci.2018;22:2817-23.

19. Huang Y, Xu Y, Lu Y, Zhu S, Guo Y, Sun C, et al.IncRNA Gm10451 regulates PTIP to facilitate iPSCsderived beta-like cell differentiation by targeting miR-338-3p as a 
ceRNA.Biomaterials.2019;216:119266.

20. Young TL, Matsuda T, Cepko CL.The noncoding RNA Taurine upregulated gene 1 is required for differentiation of the murine retina.Current Biology.2005;15:501-12.

21. Johnson R.Long non-coding RNAs in Huntington's disease neurodegeneration.Neurobiol Dis.2012;46:245-54.

22. Wu P, Zuo X, Deng H, Liu X, Liu L, Ji A.Roles of long noncoding RNAs in brain development, functional diversification and neurodegenerative diseases.Brain Res Bull.2013;97:69-80.

23. Su S, Liu J, He K, Zhang M, Feng C, Peng F, et al.Overexpression of the long noncoding RNA TUG1 protects against cold-induced injury of mouse livers by inhibiting apoptosis and inflammation.FEBS J.2016;283:1261-74.

24. Li XQ, Lv HW, Wang ZL, Tan WF, Fang B, Ma H.MiR-27a ameliorates inflammatory damage to the blood-spinal cord barrier after spinal cord ischemia: reperfusion injury in rats by downregulating TICAM-2 of the TLR4 signaling pathway.J Neuroinflammation.2015;12:25.

25. Wang Z, Liu F, Wei M, Qiu Y, Ma C, Shen L, et al.Chronic constriction injury-induced microRNA-146a$5 p$ alleviates neuropathic pain through suppression of IRAK1/TRAF6 signaling pathway.J Neuroinflammation.2018;15:179.

26. Li JJ, Wang B, Kodali MC, Chen C, Kim E, Patters BJ, et al.In vivo evidence for the contribution of peripheral circulating inflammatory exosomes to neuroinflammation.J Neuroinflammation.2018;15:8.

27. Amorim MR, de Deus JL, Cazuza RA, Mota CMD, da Silva LEV, Borges GS, et al.Neuroinflammation in the NTS is associated with changes in cardiovascular reflexes during systemic inflammation.J Neuroinflammation.2019;16:125.

28. Bao N, Fang B, Lv H, Jiang Y, Chen F, Wang Z, et al.Upregulation of miR-199a-5p Protects Spinal Cord Against Ischemia/Reperfusion-Induced Injury via Downregulation of ECE1 in Rat.Cell Mol Neurobiol.2018;38:1293-303.

29. Liu K, Yan L, Jiang X, Yu Y, Liu H, Gu T, et al.Acquired inhibition of microRNA-124 protects against spinal cord ischemia-reperfusion injury partially through a mitophagy-dependent pathway.J Thorac Cardiovasc Surg.2017;154:1498-508.

30. Hu JR, Lv GH, Yin BL.Altered microRNA expression in the ischemic-reperfusion spinal cord with atorvastatin therapy.J Pharmacol Sci.2013;121:343-6.

31. Ning B, Gao L, Liu RH, Liu Y, Zhang NS, Chen ZY.microRNAs in spinal cord injury: potential roles and therapeutic implications.Int J Biol Sci.2014;10:997-1006.

32. Meza-Sosa KF, Valle-Garcia D, Pedraza-Alva G, Perez-Martinez L.Role of microRNAs in central nervous system development and pathology.J Neurosci Res.2012;90:1-12.

33. Saito Y, Saito H.MicroRNAs in cancers and neurodegenerative disorders.Front Genet.2012;3:194.

34. Wei R, Zhang R, Li H, Li H, Zhang S, Xie Y, et al.MiR-29 Targets PUMA to Suppress Oxygen and Glucose Deprivation/Reperfusion (OGD/R)-induced Cell Death in Hippocampal Neurons.Curr 
Neurovasc Res.2018;15:47-54.

35. Reinhold AK, Rittner HL.Barrier function in the peripheral and central nervous system-a review.Pflugers Arch.2017;469:123-34.

36. Li XQ, Cao XZ, Wang J, Fang B, Tan WF, Ma H.Sevoflurane preconditioning ameliorates neuronal deficits by inhibiting microglial MMP-9 expression after spinal cord ischemia/reperfusion in rats.Mol Brain.2014;7:69.

37. Li XQ, Wang J, Fang B, Tan WF, Ma H.Intrathecal antagonism of microglial TLR4 reduces inflammatory damage to blood-spinal cord barrier following ischemia/reperfusion injury in rats.Mol Brain.2014;7:28.

38. Vartak-Sharma N, Gelman BB, Joshi C, Borgamann K, Ghorpade A.Astrocyte elevated gene-1 is a novel modulator of HIV-1-associated neuroinflammation via regulation of nuclear factor-kappaB signaling and excitatory amino acid transporter-2 repression.J Biol Chem.2014;289:19599-612.

39. Wang Y, Zhang W, Zhu X, Wang Y, Mao X, Xu X, et al.Upregulation of AEG-1 Involves in Schwann Cell Proliferation and Migration After Sciatic Nerve Crush.J Mol Neurosci.2016;60:248-57.

40. Zhang W, Bi Y, Li J, Peng F, Li H, Li C, et al.Long noncoding RNA FTX is upregulated in gliomas and promotes proliferation and invasion of glioma cells by negatively regulating miR-342-3p.Lab Invest.2017;97:447-57.

41. Su Q, Liu Y, Lv XW, Ye ZL, Sun YH, Kong BH, et al.Inhibition of IncRNA TUG1 upregulates miR-142-3p to ameliorate myocardial injury during ischemia and reperfusion via targeting HMGB1- and Rac1induced autophagy.J Mol Cell Cardiol.2019;133:12-25.

42. Liang Z, Ren C.Emodin attenuates apoptosis and inflammation induced by LPS through upregulating IncRNA TUG1 in murine chondrogenic ATDC5 cells.Biomed Pharmacother.2018;103:897902.

43. Bao MH, Szeto V, Yang BB, Zhu SZ, Sun HS, Feng ZP.Long non-coding RNAs in ischemic stroke.Cell Death Dis.2018;9:281.

44. Chen S, Wang M, Yang H, Mao L, He Q, Jin H, et al.LncRNA TUG1 sponges microRNA-9 to promote neurons apoptosis by up-regulated Bcl2/11 under ischemia.Biochem Biophys Res Commun.2017;485:167-73.

45. Wu ZW, Zhao SJ, Li CF, Liu CQ.LncRNA TUG1 serves an important role in hypoxia-induced myocardial cell injury by regulating the miR-145-5p-Binp3 axis.Molecular Medicine Reports.2018;17:2422-30.

46. He Y, Li M, Wujisiguleng, Lv B, Huan Y, Liu B, et al.Zhenbao Pill reduces Treg cell proportion in acute spinal cord injury rats by regulating TUG1/miR-214/HSP27 axis.Biosci Rep.2018;38.

47. Schmitz SU, Grote P, Herrmann BG.Mechanisms of long noncoding RNA function in development and disease.Cell Mol Life Sci.2016;73:2491-509.

48. Qiao Y, Peng C, Li J, Wu D, Wang X.LncRNA MALAT1 is Neuroprotective in a Rat Model of Spinal Cord Ischemia-Reperfusion Injury Through miR-204 Regulation.Curr Neurovasc Res.2018;15:211-19. 
49. Liu Y, Pan L, Jiang A, Yin M.Hydrogen sulfide upregulated IncRNA CasC7 to reduce neuronal cell apoptosis in spinal cord ischemia-reperfusion injury rat.Biomed Pharmacother.2018;98:856-62.

50. Liu B, Teschemacher AG, Kasparov S.Neuroprotective potential of astroglia.J Neurosci Res.2017;95:2126-39.

51. Li G, Wang Z, Ye J, Zhang X, Wu H, Peng J, et al.Uncontrolled inflammation induced by AEG-1 promotes gastric cancer and poor prognosis.Cancer Res.2014;74:5541-52.

52. Fang Y, Shen H, Cao Y, Li H, Qin R, Chen Q, et al.Involvement of miR-30c in resistance to doxorubicin by regulating YWHAZ in breast cancer cells.Braz J Med Biol Res.2014;47:60-9.

\section{Figures}

a

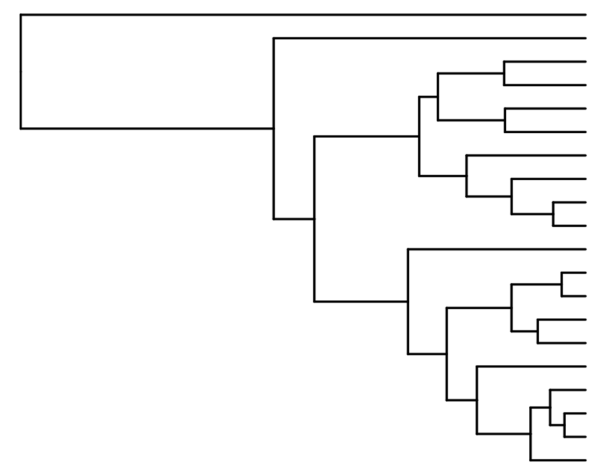

Color Key

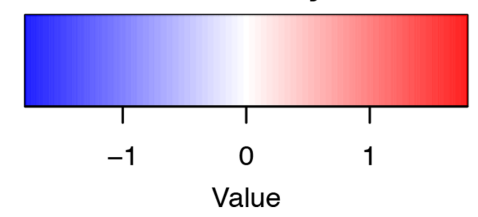

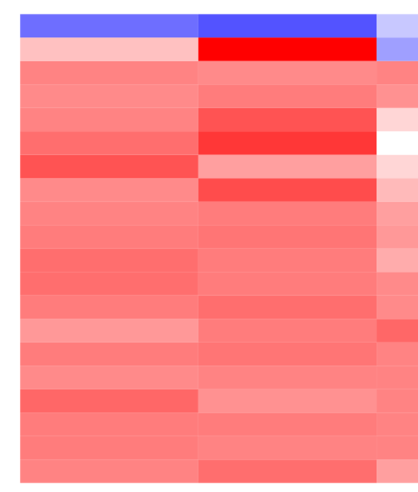

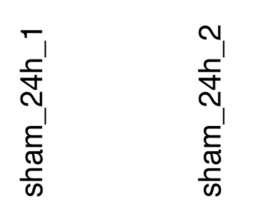

rno-miR-3568 rno-miR-872-3p rno-miR-338-3p rno-miR-181a-5p rno-miR-328b-3p rno-miR-702-3p rno-miR-410-5p rno-miR-181a-1-3p rno-miR-488-3p rno-miR-221-3p rno-miR-181b-1-3p rno-miR-135b-5p rno-miR-129-1-3p rno-miR-129-1-3p
rno-miR-9a-5p $\frac{\text { rno-miR-29b-1-5p }}{\text { rno-miR-196b-5p }}$ rno-miR-196b-5p
rno-miR-582-3p rno-miR-582-3p rno-miR-879-3p rno-miR-370-5p

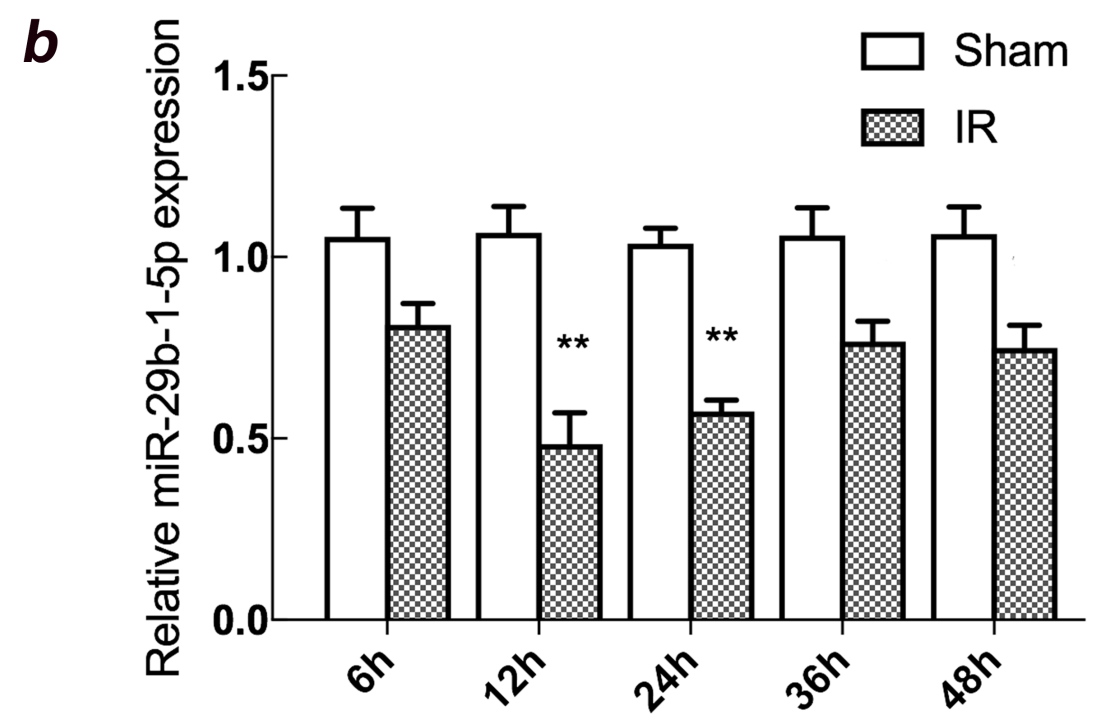

Figure 1 
Aberrant miR-29b-1-5p expression after IR in rat spinal cord. (a) Heatmap showing aberrantly expressed miRNAs ( $n=3$ per group). (b) MiR-29b-1-5p expression levels by qRT-PCR ( $n=3$ per group). The data are expressed as means \pm SEM. ${ }^{* *} p<0.05$ compared with the sham.
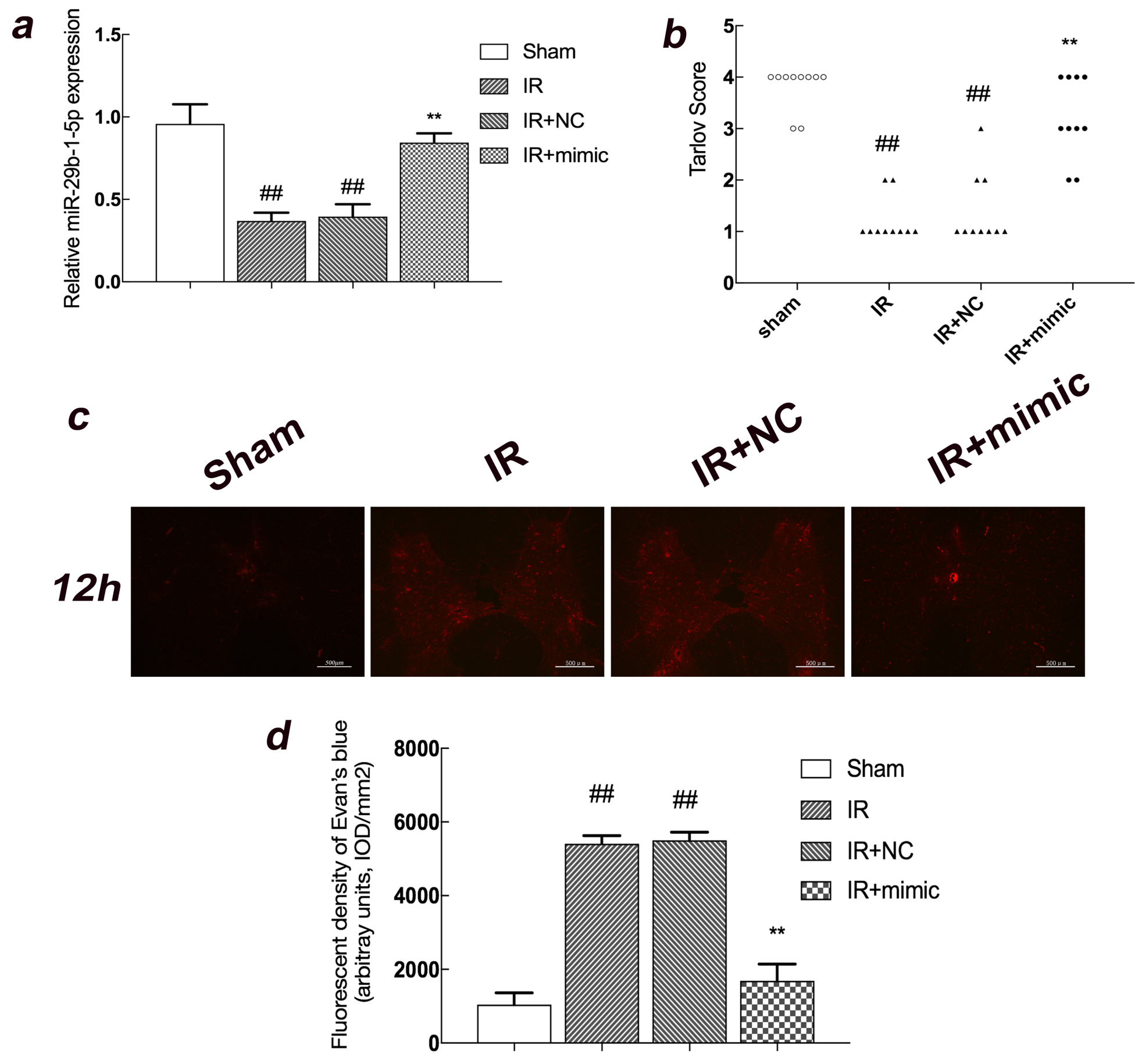

Figure 2

Mimic-29b-1-5p attenuated neurological defects and BSCB leakage after IR. (a) MiR-29b-1-5p expression in rat spinal cord ( $n=3$ per group). (b) Tarlov scores in the indicated groups ( $n=10$ per group). (c) Representative images of spinal cord sections showing EB fluorescence. (d) Fluorescence density of EB $(\mathrm{IOD} / \mathrm{mm} 2)$ in the indicated groups. Scale bar $=500 \mu \mathrm{m}, \mathrm{n}=3$ per group. Data are expressed as means \pm SEM. \#\#p $<0.05$ compared to sham; $*$ $p<0.05$ compared to IR. 
a Rat MTDH(165-171) 5'...AAAGGAAAGUUAAUUUAUGAAAU....3'

Rat miR-29b-1-5p 3 '-UUUAGAUUUGgUGGUAUACUUU-5'

b Rat TRIL(562-568) 5'...AAGCUACCUGAGACAAUAUGAaG...3'

C

TRIL

Sham IR

MTDH

B-actin

Rat miR-29b-1-5p 3'-UUUAGAUUUGGUGGUAUACUUU-5'
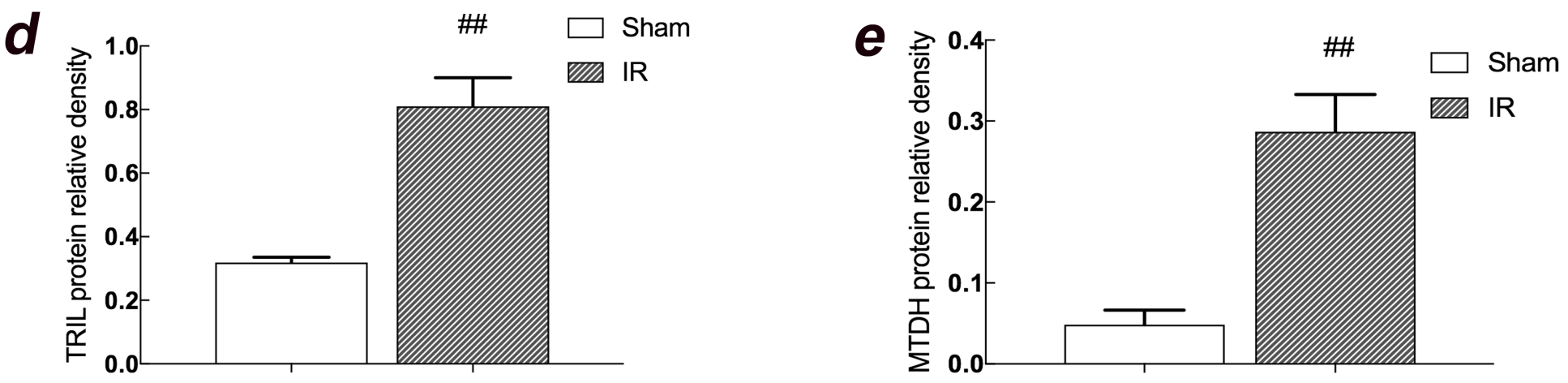

Figure 3

MTDH is the target of miR-29b-1-5p. (a) Predicted miR-29b-1-5p and MTDH binding sites. (b) Predicted miR-29b-1-5p and TRIL binding sites. (c) Immunoblot showing TRIL and MTDH protein levels in rat spinal cord. (d-e) Densities of TRIL and MTDH bands normalized to $\beta$-actin ( $n=3$ per group). Data are expressed as means \pm SEM. \#\#p $<0.05$ compared to sham.

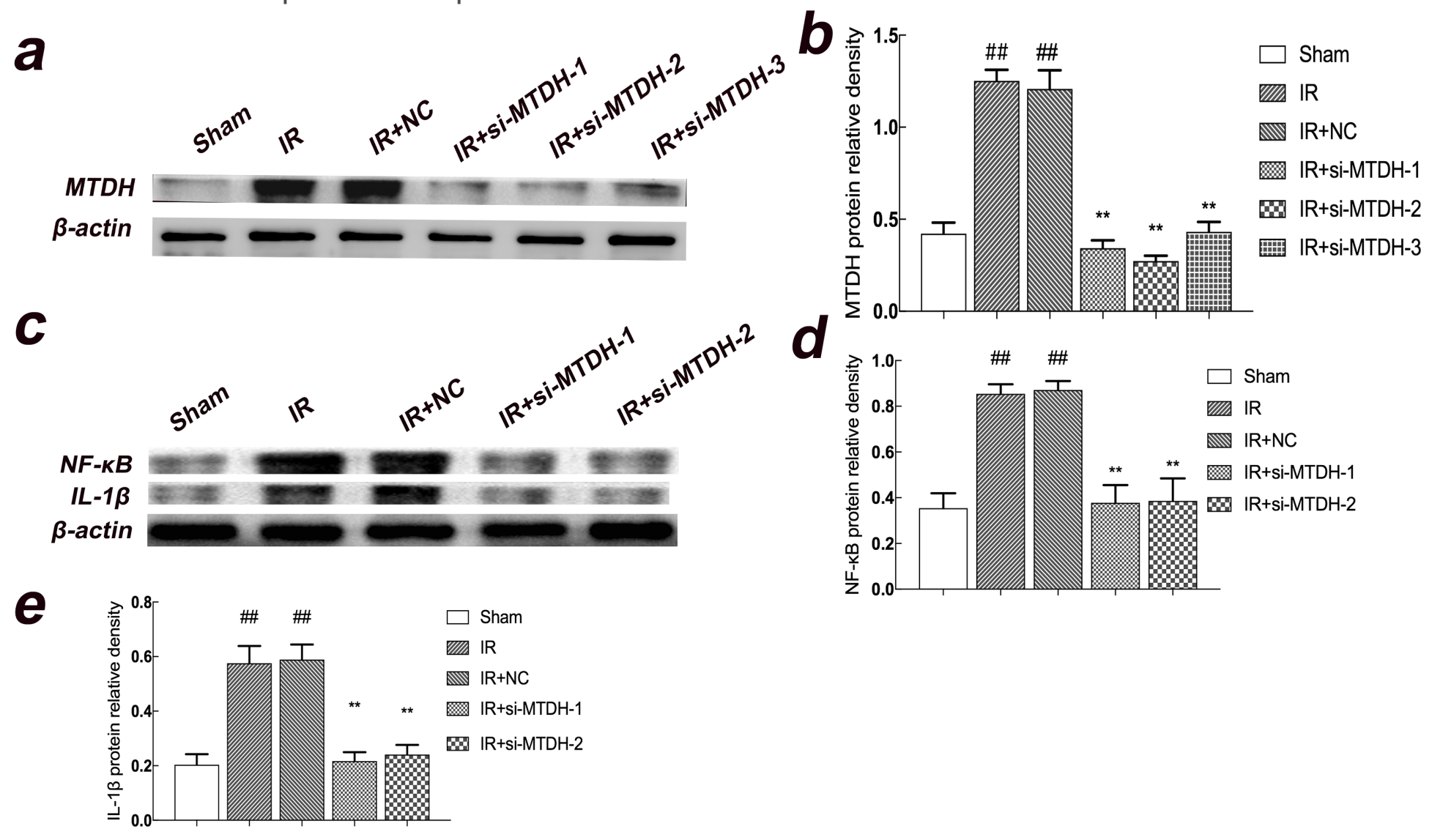


Figure 4

MTDH knockdown inhibited the NF-KB pathway. (a) Immunoblot showing MTDH expression in rat spinal cord. (b) Densities of MTDH bands normalized to $\beta$-actin ( $n=3$ per group). (c) Immunoblot showing NFKB p65 and IL-1 $\beta$ levels in rat spinal cord. (d, e) Densities of NF-KB p65 and IL-1 $\beta$ bands normalized to $\beta$ actin $(n=3$ per group). Data are expressed as means \pm SEM. \#\#p $<0.05$ compared to sham, $* \star p<0.05$ compared to IR.

a

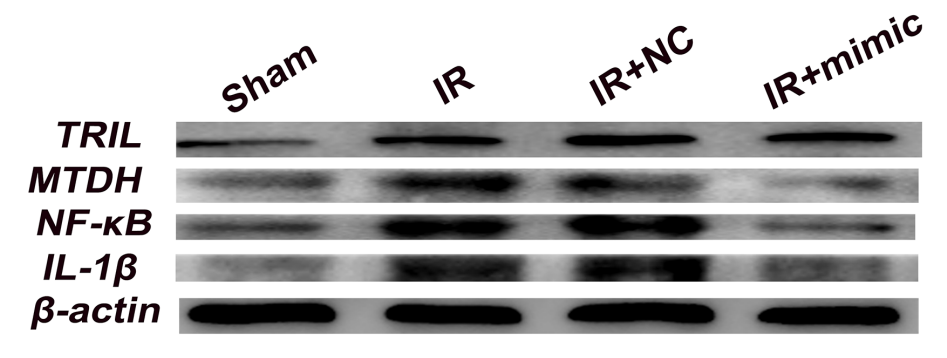

C

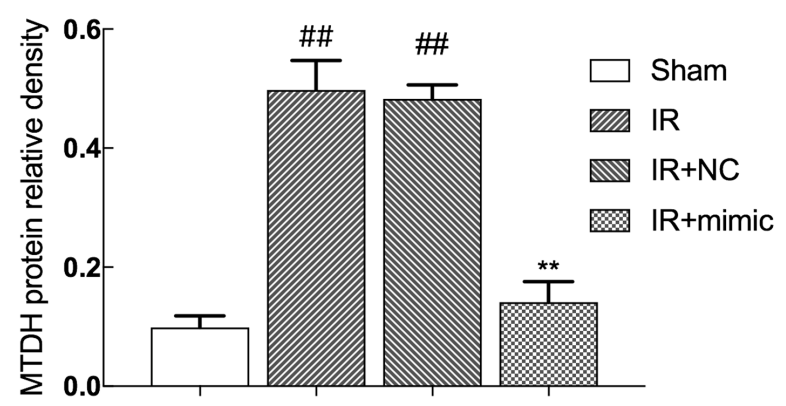

e

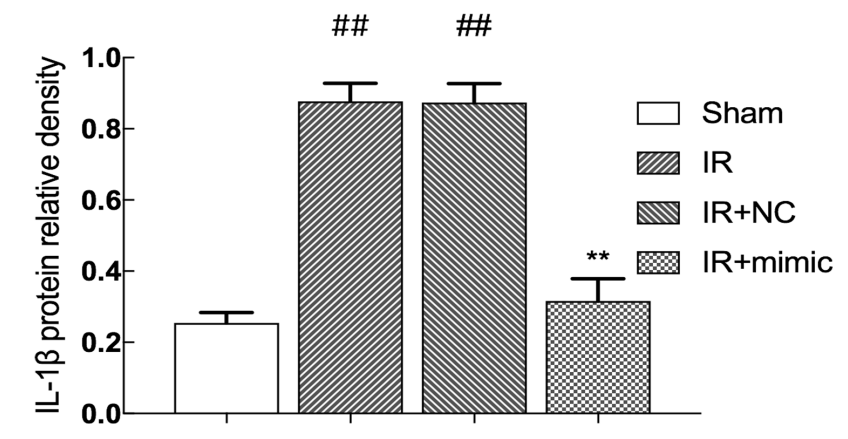

g

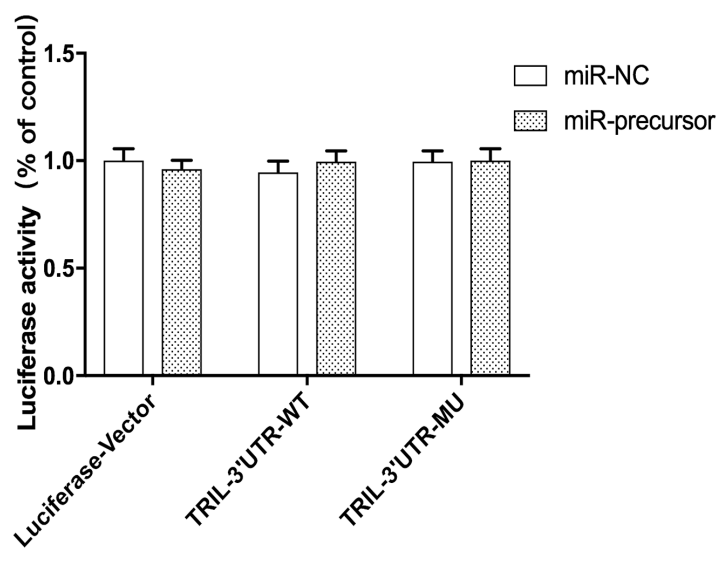

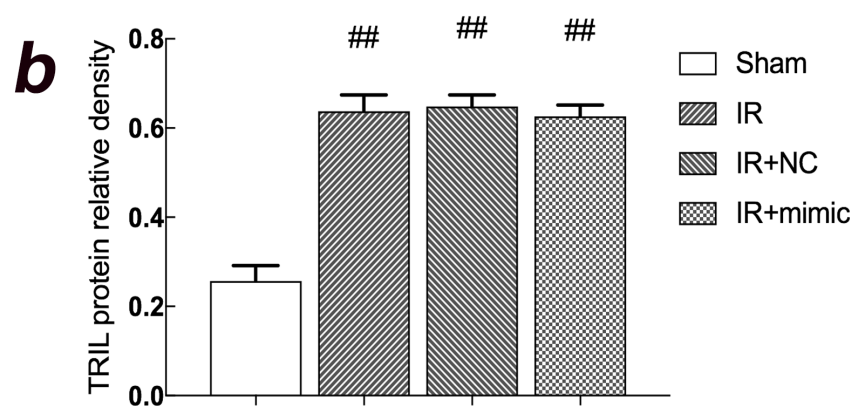
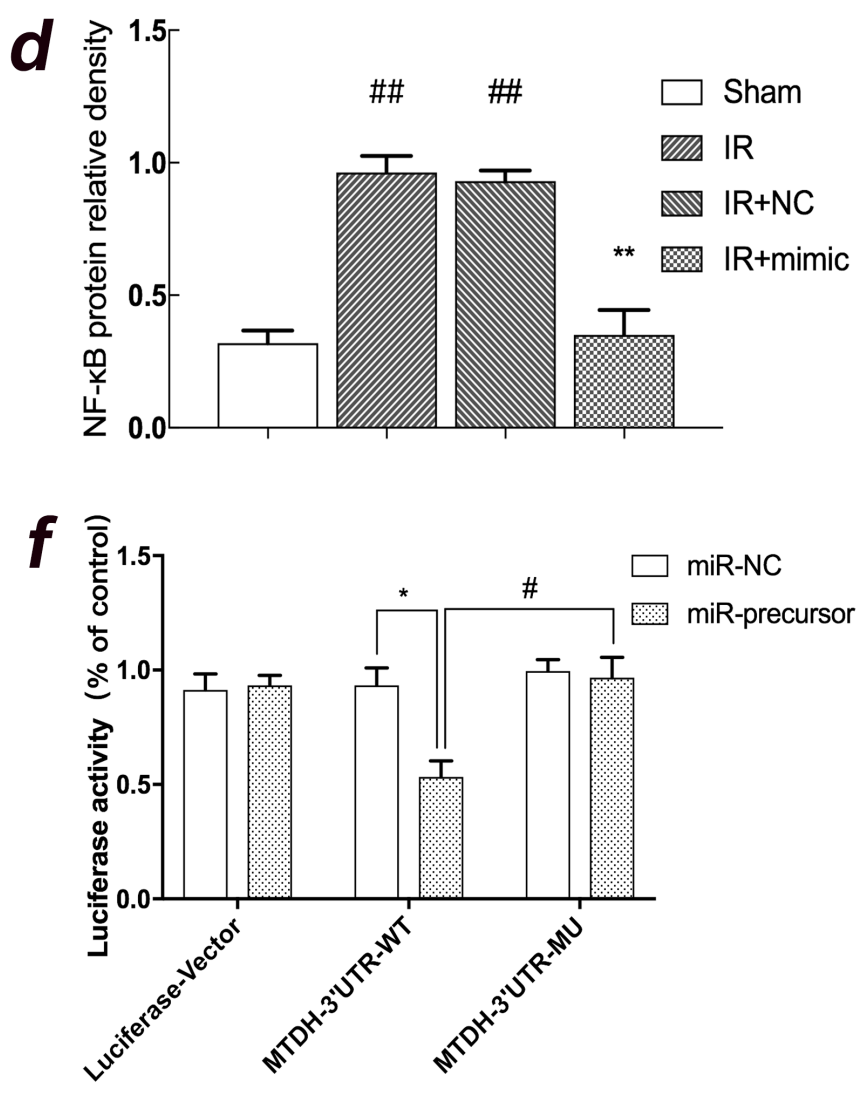

Figure 5 
Mimic-miR-29b-1-5p inhibited MTDH/NF-KB/IL-1 $\beta$ after IR. (a) Immunoblot showing TRIL, MTDH, NF-KB and IL-1 $\beta$ expression in rat spinal cord. (b-e) Densities of TRIL, MTDH, NF-KB and IL-1 $\beta$ bands normalized to $\beta$-actin ( $n=3$ per group). Data are expressed as means \pm SEM. \#\#p $<0.05$ compared to sham; ${ }^{* \star} p<$ 0.05 compared to IR. (f) Dual-luciferase reporter assay using miR-29b-1-5p and MTDH-3'-UTR constructs. (g) Dual-luciferase reporter assay using miR-29b-1-5p and TRIL-3'-UTR constructs. Data are expressed as means \pm SEM. * $p<0.05$ vs. MTDH -WT+ miR-29b-1-5p NC; \#p < 0.05 vs. MTDH-MU+ miR-29b-1-5p precursor.

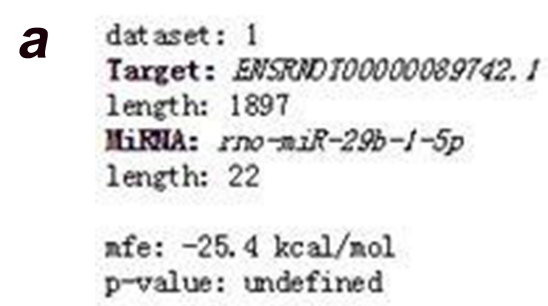

a dataset: 1

Target: ENSSNDT00000089742. I

length: 1897

HiFIA: $r n o-x i R-29 b-1-5 p$

length: 22

afe: $-25.4 \mathrm{kcal} / \mathrm{nol}$

p-value: undefined

Position: 1313

target 5' C UU G U 3' AUCU AOCACCAU GUGA

UWGA UGGUGGUA UACU

niRNs 3 UU UU UU 5 ,

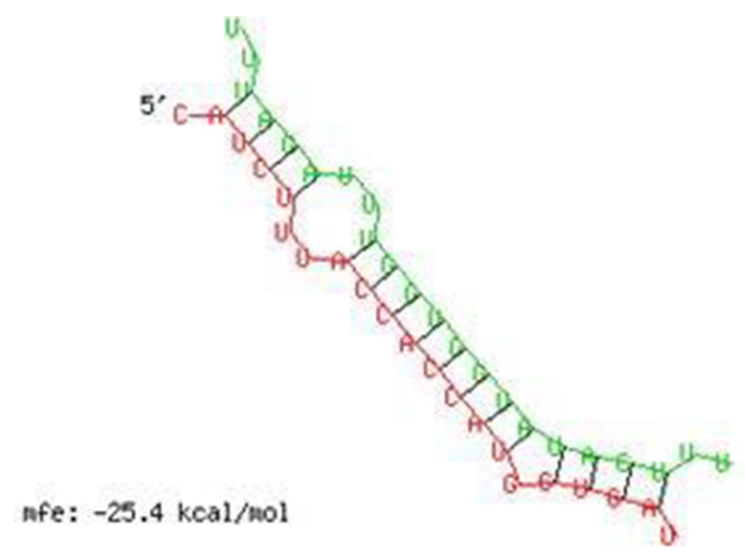

b

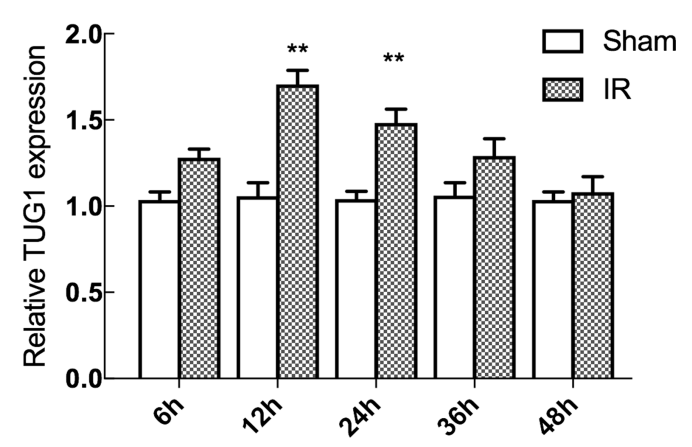

d

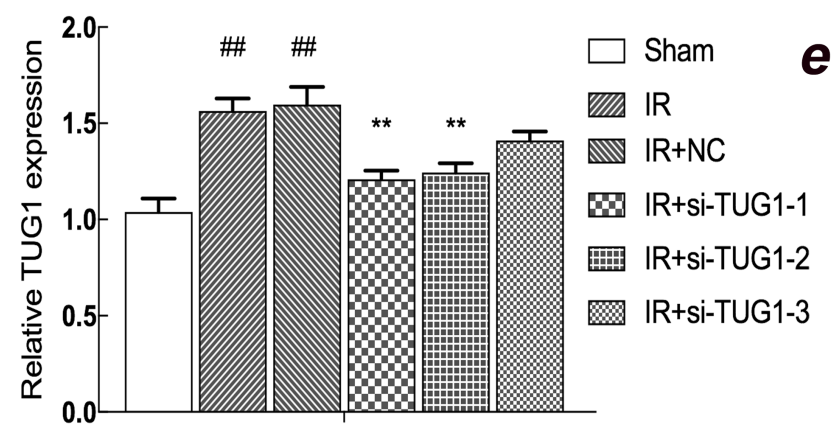

f

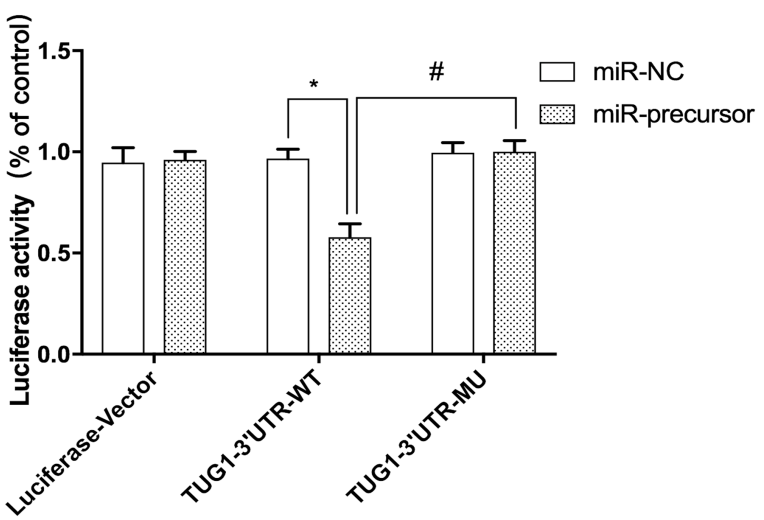

C

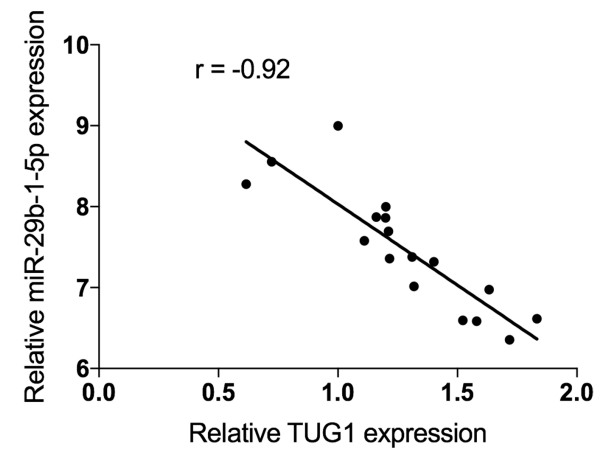

e

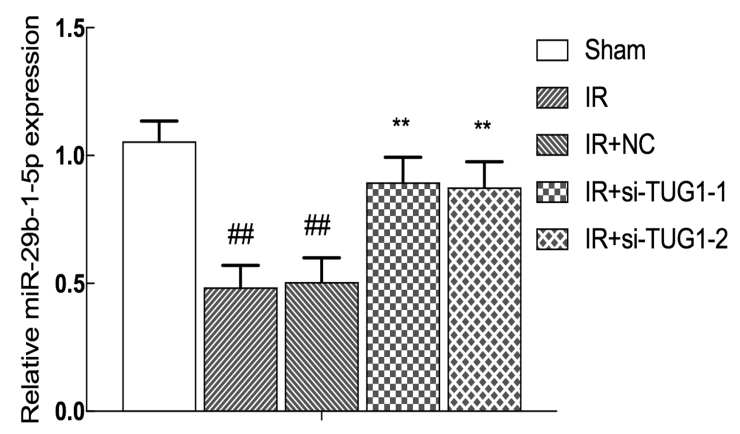


Figure 6

TUG1 knockdown upregulated miR-29b-1-5p after IR. (a) Predicted miR-29b-1-5p and TUG1 binding sites.

(b) TUG1 expression levels in rat spinal cord ( $n=3$ per group). Data are expressed as means $\pm S E M . * \star p<$ 0.05 compared to sham. (c) Correlation between TUG1 and miR-29b-1-5p ( $n=20$ total), $p<0.001$, and levels of (d) TUG1 and (e) miR-29b-1-5p in the spinal cord ( $n=3$ per group). Data are expressed as means \pm SEM. \#\#p $<0.05$ compared to sham; ${ }^{\star *} p<0.05$ compared to IR. (f) Dual-luciferase reporter assay using TUG1 and miR-29b-1-5p constructs. ${ }^{*} p<0.05$ vs. TUG1-WT+ miR-29b-1-5p NC group; \#p< 0.05 vs. TUG1MU+ miR-29b-1-5p precursor group.

a

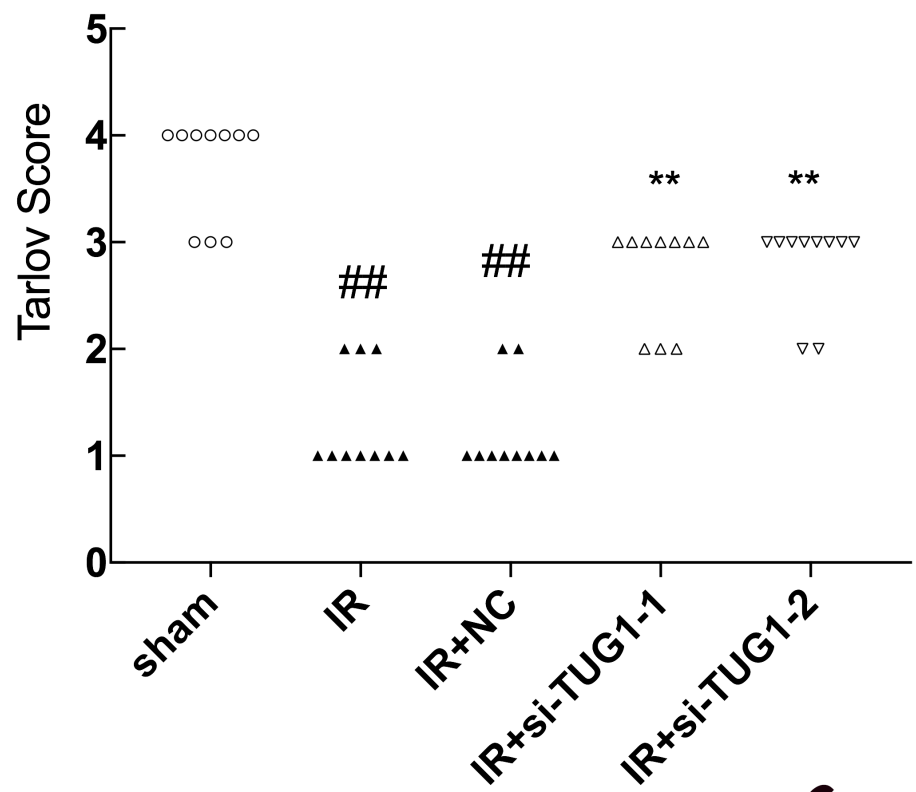

b
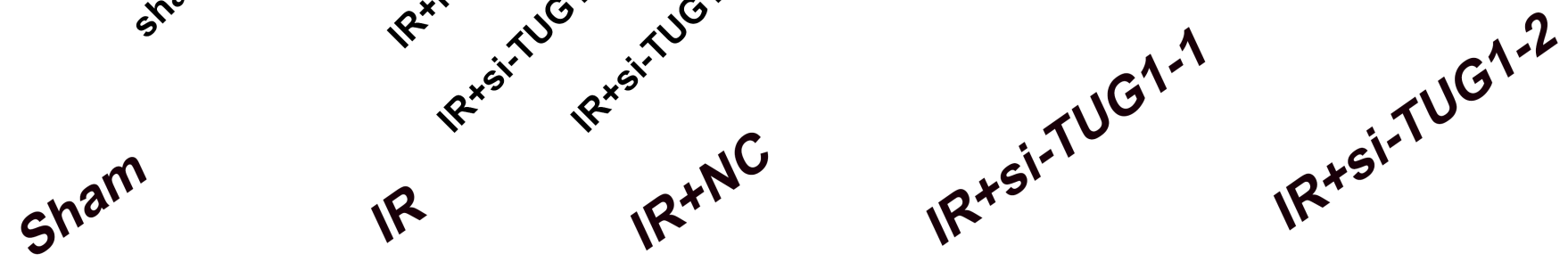

$12 h$
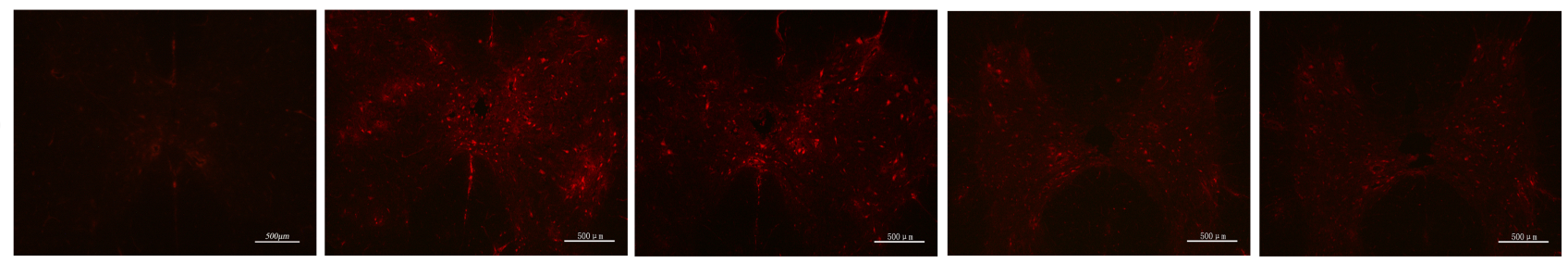

c

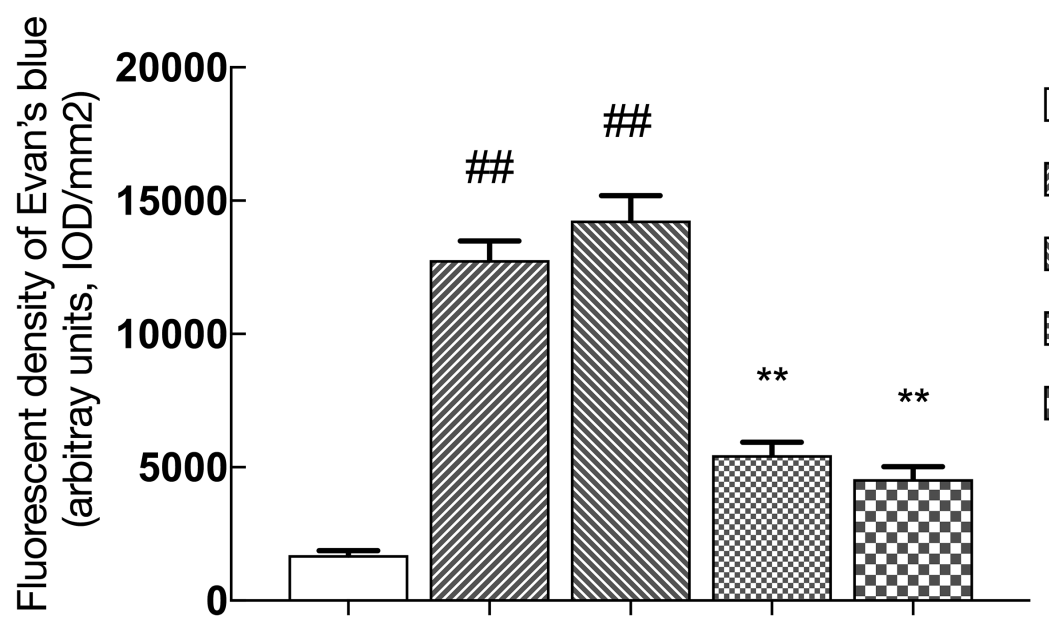


Figure 7

TUG1 knockdown relieved IR-induced neurological defects and BSCB leakage. (a) Tarlov scores in the indicated groups ( $n=10$ per group). (b) Representative images showing EB fluorescence in the spinal cord sections. (c) Fluorescence intensity of EB $(I O D / \mathrm{mm} 2)$ in the indicated groups. Scale bar $=500 \mu \mathrm{m}, \mathrm{n}$ $=3$ per group. Data are expressed as means \pm SEM. \#\#p $<0.05$ compared to sham; ${ }^{\star \star} p<0.05$ compared to IR.

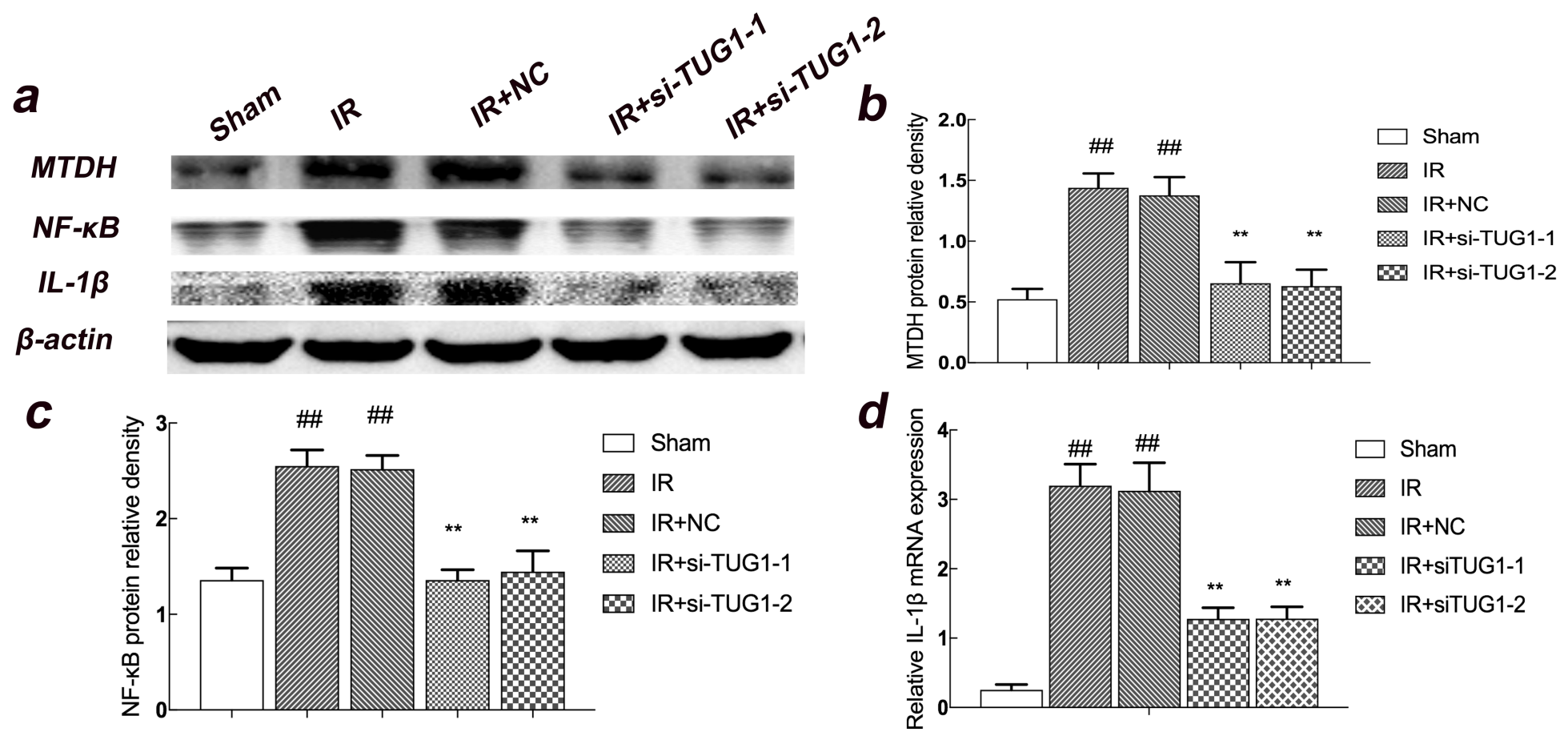

Figure 8

TUG1 knockdown promotes MTDH/NF-KB/IL-1 $\beta$ pathway after IR. (a) Immunoblot showing MTDH, NF-KB and IL-1 $\beta$ in rat spinal cord. (b-d) Densities of MTDH, NF-KB and IL-1 $\beta$ bands normalized to $\beta$-actin ( $n=3$ per group). Data are expressed as means \pm SEM. \#\#p $<0.05$ compared to sham; ${ }^{* \star} p<0.05$ compared to IR. 
a

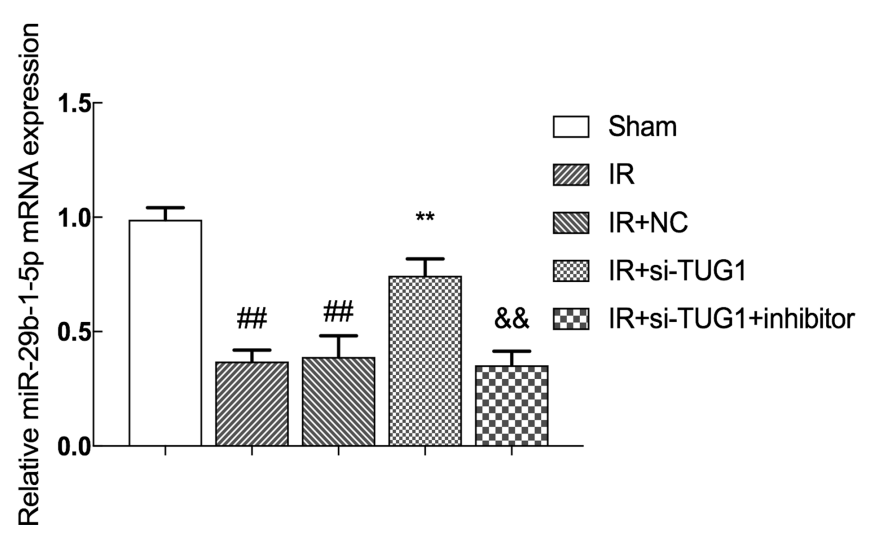

b

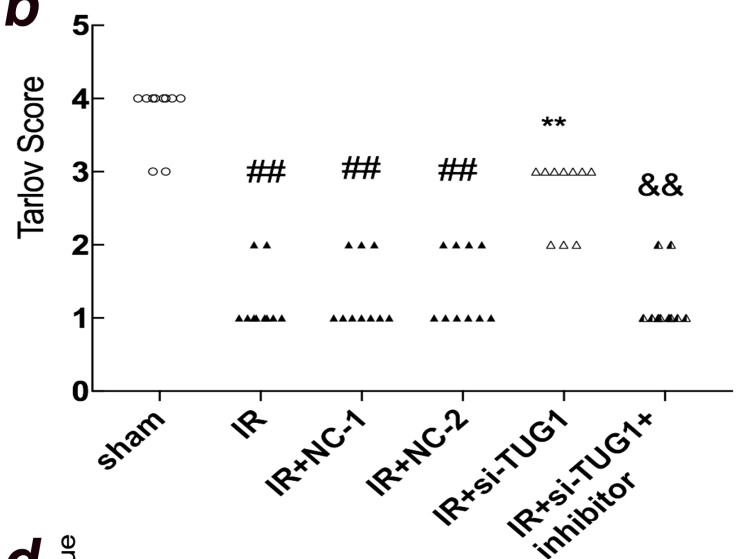

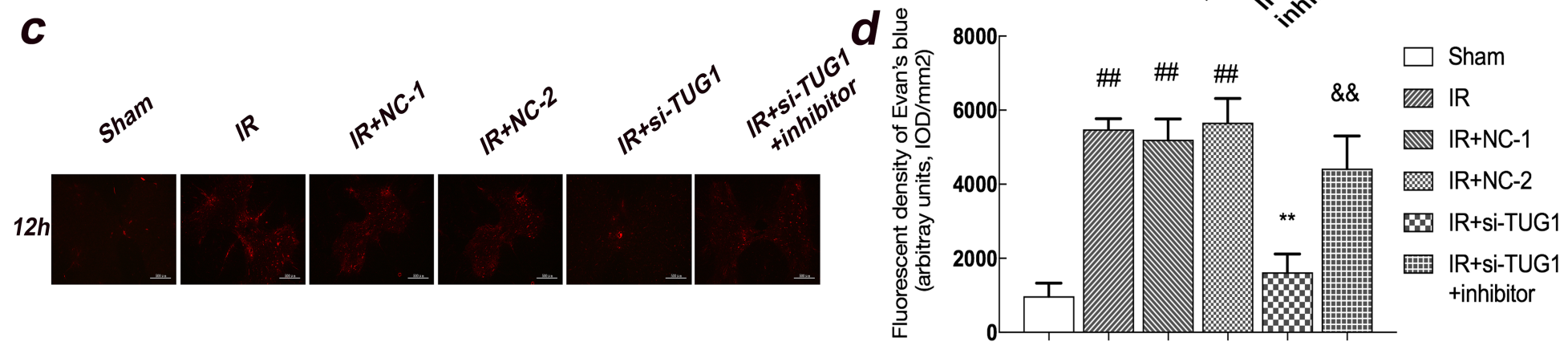

Figure 9

TUG1 and miR-29b-1-5p interaction in spinal cord IR. (a) MiR-29b-1-5p expression in rat spinal cord ( $\mathrm{n}=3$ per group). (b) Tarlov scores in the indicated groups ( $n=10$ per group). (c) Representative images of spinal cord sections showing EB fluorescence (d) Fluorescence intensities of EB (IOD/mm2) in the indicated groups. Scale bar $-500 \mu \mathrm{m}, \mathrm{n}=3$ per group. Data are expressed as means $\pm S E M$. \#\#p $<0.05$ compared to sham; ${ }^{\star \star} \mathrm{p}<0.05$ compared to IR; \&\&p $<0.05$ compared to IR+si-TUG1. 


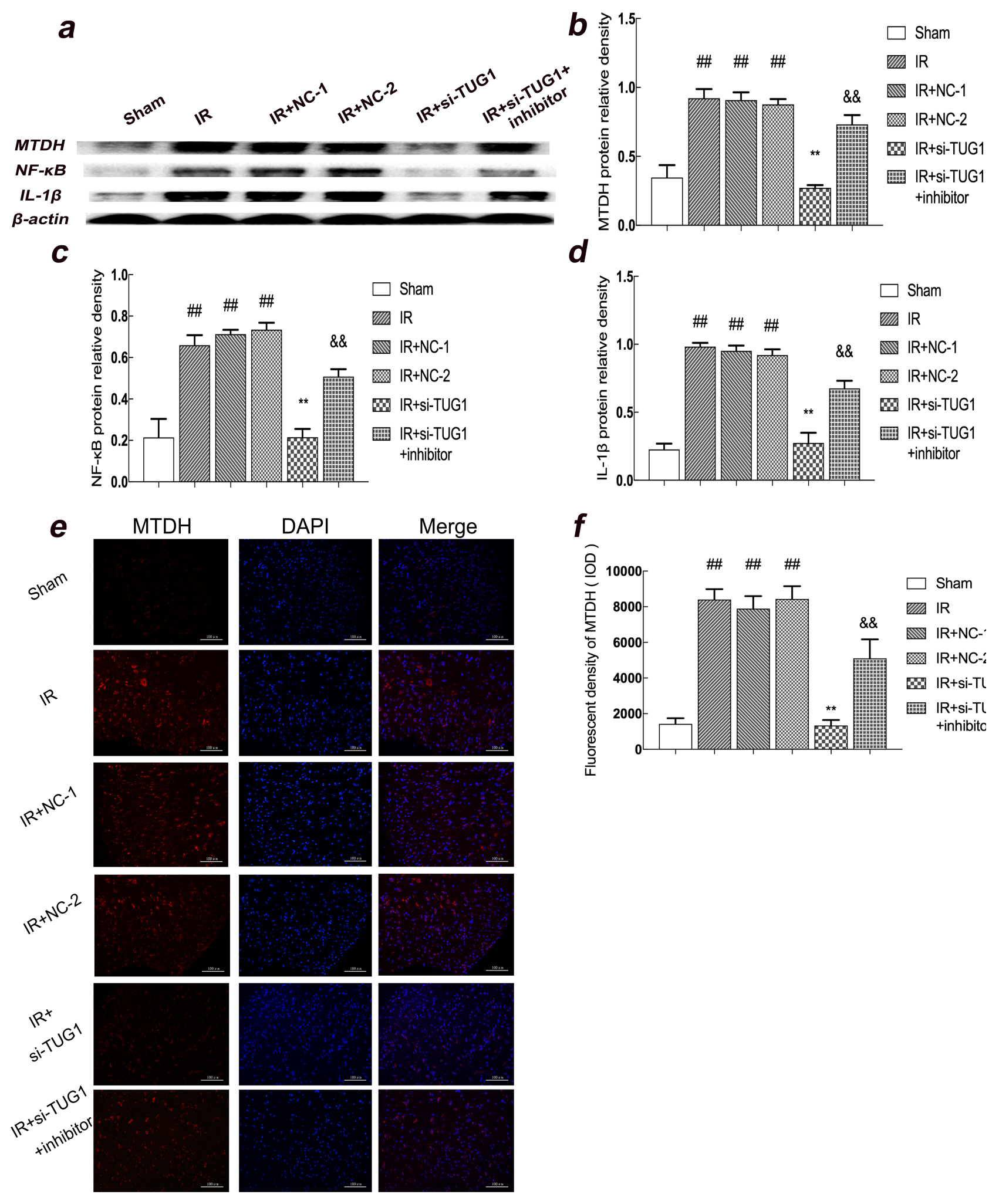

Figure 10

MiR-29-1-5p inhibitor restores the MTDH/NF-KB/IL-1 $\beta$ pathway after IR. (a) Immunoblot showing MTDH, NF-KB, and IL-1 $\beta$ expression in rat spinal cord. (b-d) Densities of MTDH, NF-KB and IL-1 $\beta$ bands normalized to $\beta$-actin ( $n=3$ per group). (e) Representative images showing co-localization of MTDH (red) and DAPI (blue) in rat spinal cord. Scale bars - $100 \mu \mathrm{m}$. (f) Fluorescence intensities of MTDH (IOD) in the 
indicated groups ( $\mathrm{n}=3$ per group). Data are expressed as means $\pm S E M$. \#\#p $<0.05$ compared to sham; ** $\mathrm{p}<0.05$ compared to IR; \&\&p $<0.05$ compared to IR+si-TUG1.

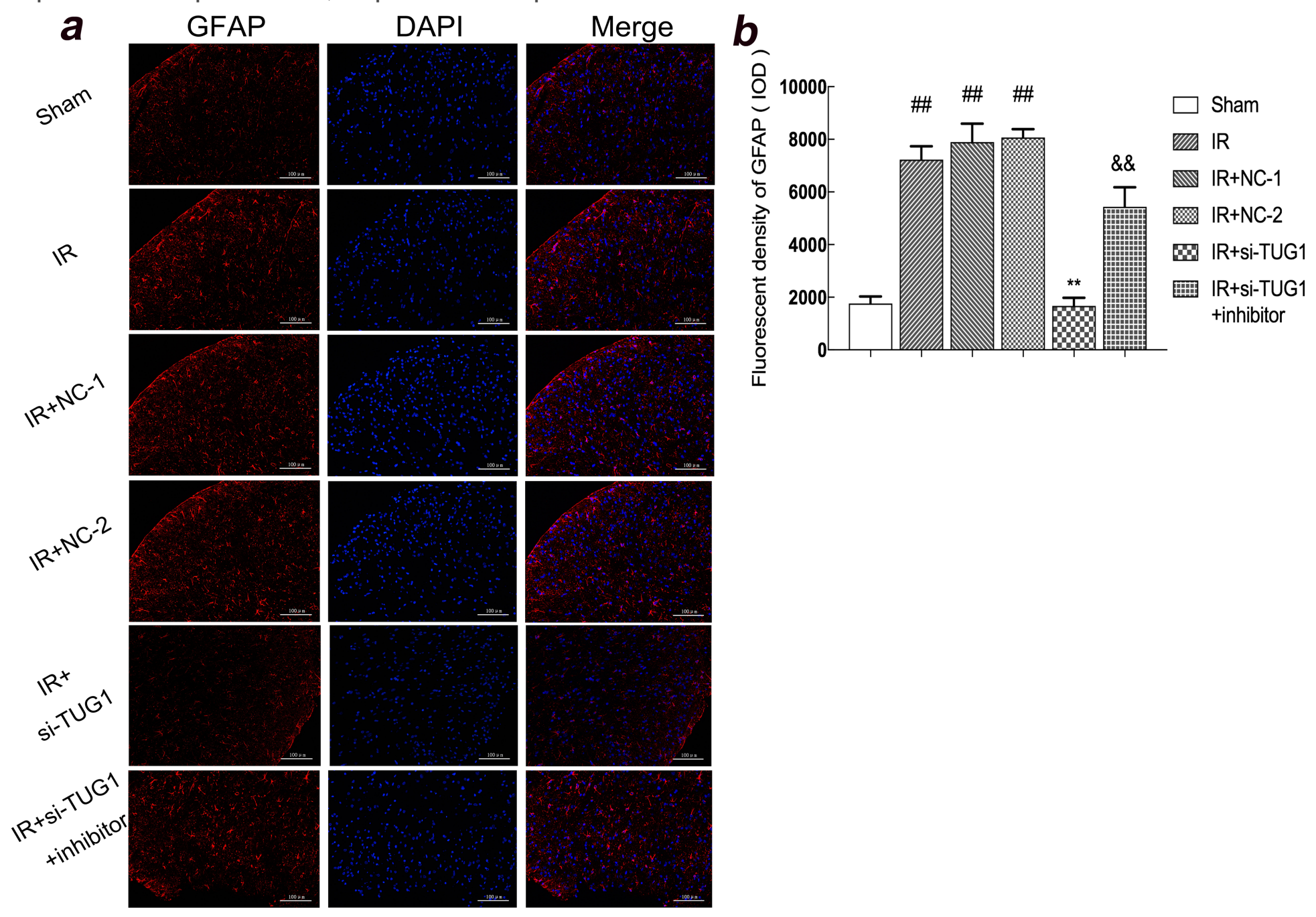

\section{Figure 11}

TUG1 knockdown increases astrogliosis after IR. (a) Representative images showing co-localization of

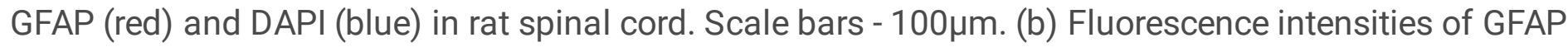
(IOD) in the indicated groups ( $n=3$ per group). Data are expressed as means \pm SEM. \#\#p $<0.05$ compared to sham; ${ }^{* *} \mathrm{p}<0.05$ compared to IR; \&\&p $<0.05$ compared to IR+si-TUG1. 